\title{
Nonlinear Vibrations of a Mechanism for the Miller-Atkinson Cycle
}

\author{
Ionuţ Dragomir ${ }^{1}$, Bogdan Mănescu ${ }^{1}$, Nicolae-Doru Stănescu ${ }^{2, *}$ \\ ${ }^{1}$ AKKA ROMSERV, Bucharest, Romania \\ ${ }^{2}$ University of Piteşti, Piteşti, Romania
}

\begin{abstract}
The distribution mechanism for the Miller-Atkinson is presented based on some previous papers of the authors. The cam is obtained in an analytical way and the optimization procedure of its shape based on Jarvis March algorithm is also described. The non-linear vibrations of the mechanism are analytically obtained used the Fourier transformation and they are numerically validated for different types of cams.
\end{abstract}

\section{Introduction}

The references were discussed in our previous papers [911] and will not repeat here. The most of the scientists usually study the phenomena in an engine working after the Miller-Atkinson cycle. The study of the distribution mechanism is a very poor one because of its complexity and of the fact that the obtained cam is not a convex one. In our paper we will use the Jarvis March in order to obtain a convex cam and we will compare the results obtained in classical way with those deduced for the convex cam.

The distribution mechanism considered in our paper is presented in previous works of the authors [9-11]. It consists in a valve moving on the vertical direction (Figs. 1-4), a lever $O B$ rotating around the revolute joint situated at the point $O$, at the end of the lever being situated a roll of radius $R_{2}$. The lever is rigidly linked to the bar $O D$, at the end $D$ of which being situated another roll of radius $R_{3}$ (Fig. 5). The cam of center $O$ (Fig. 5) rotates around its center, rotating the element $\mathrm{DO}_{2} \mathrm{~B}$ and acting the valve.

\section{Synthesis of cam}

There exist four different variants to be considered:

i) the valve is asymmetrically acted, the head of valve being a spherical one;

ii) the valve is symmetrically acted, the head of valve being a spherical one;

iii) the valve is asymmetrically acted, the head of valve being a planar one;

iv) the valve is symmetrically acted, the head of valve being a planar one.

In the case i) one knows the maximum displacement of the valve $s_{\max }$, its law of motion $s=s(\varphi)$, the initial angle $\beta_{0}$ for which it results $\theta=0$. The radius of the spherical head of valve is equal to $R_{1}$. The lever $O D$ (Fig. 1) has a length equal to $l_{1}$. A displacement $s$ of the valve leads to a rotation of angle $\theta$ for the lever.

a)

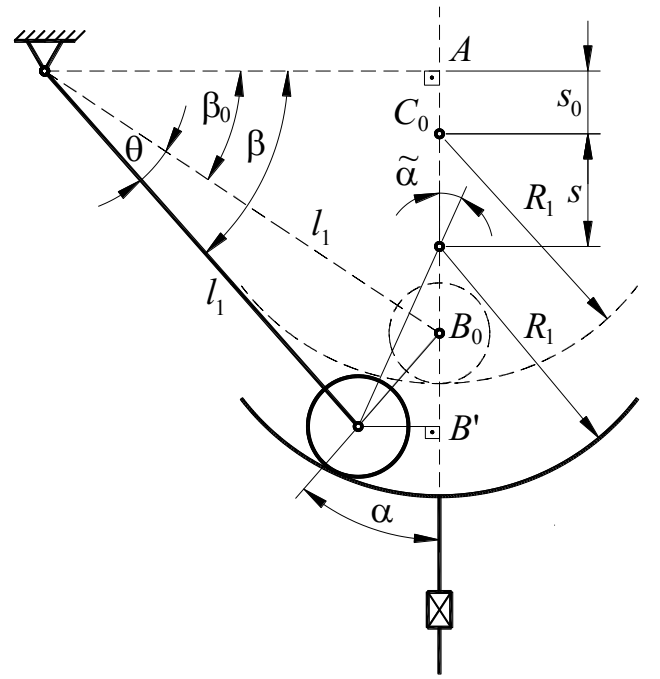

b)

Fig. 1. The mechanism in the asymmetric case: $a$ ) analytic approach; b) geometric approach.

An analytic approach (Fig. 1, a)) offers [9-11] the implicit equation from which one may obtain the rotational angle $\beta$

$$
\begin{gathered}
\left\{2 x_{C}\left(l_{1} \sin \beta-y_{C}\right)^{2}+\left(l_{1} \cos \beta-x_{C}\right)\right. \\
\left.\times\left[C_{1}-2 y_{C}\left(l_{1} \sin \beta-y_{C}\right)\right]\right\} \\
-\left(l_{1}^{2}+x_{C}^{2}+y_{C}^{2}-2 l_{1} x_{C} \cos \beta-2 l_{1} y_{C} \sin \beta\right) \\
\times\left\{4 x_{C}^{2}\left(l_{1} \sin \beta-y_{C}\right)^{2}\right. \\
+\left[C_{1}-2 y_{C}\left(l_{1} \sin \beta-y_{C}\right)\right]^{2} \\
\left.-4 R_{1}^{2}\left(l_{1} \sin \beta-y_{C}\right)\right\}=0,
\end{gathered}
$$

\footnotetext{
* Corresponding author: s_doru@yahoo.com,doru.stanescu@upit.ro
} 
where the parameters $C_{1}, x_{C}$ and $y_{C}$ have the following expressions $C_{1}=R_{1}^{2}-R_{2}^{2}+l_{1}^{2}-\left(x_{C}^{2}+y_{C}^{2}\right)$, $x_{C}=d, y_{C}=h+s-R_{1}$.

Following a geometric approach (Fig. 1, b)) one obtain the relation

$$
\begin{aligned}
& -s+l_{1}\left[\sin \left(\theta+\beta_{0}\right)-\sin \beta_{0}\right] \\
& +\left(R_{1}-R_{2}\right)(1-\cos \tilde{\alpha})=0,
\end{aligned}
$$

where

$$
\widetilde{\alpha}=\arcsin \left(\frac{l_{1}\left[\cos \beta_{0}-\cos \left(\beta_{0}+\theta\right)\right]}{R_{1}-R_{2}}\right) .
$$

For the situation described in ii) (Fig. 2) one may write [11] the geometric relations

$$
\begin{gathered}
\beta_{0}=\arccos \left(\frac{d_{1}+d_{2}}{l_{1}}\right), \tilde{\alpha}_{0}=\arcsin \left(\frac{d_{2}}{R_{1}}\right), \\
\alpha=\beta_{0}+\frac{\theta}{2}, B_{0} B=\tilde{d}=2 l_{1} \sin \left(\frac{\theta}{2}\right), \\
\sin \tilde{\alpha}=\frac{d_{2}-\tilde{d} \sin \alpha}{R_{1}}, \\
\tilde{\alpha}=\arcsin \left(\frac{d_{2}-2 l_{1} \sin \alpha \sin \left(\frac{\theta}{2}\right)}{R_{1}}\right), \\
C B^{\prime}=R_{1} \cos \widetilde{\alpha}_{,} C_{0} B^{\prime}=R_{1} \cos \tilde{\alpha}_{0}+\tilde{d} \cos \alpha, \\
s=C_{0} B^{\prime}-C B^{\prime}, \\
s=R_{1} \cos \tilde{\alpha}_{0}-R_{1} \cos \tilde{\alpha}+2 l_{1} \cos \alpha \sin \left(\frac{\theta}{2}\right), \\
\tilde{\alpha}_{\text {final }}=\tilde{\alpha}_{f}=-\alpha_{0}
\end{gathered}
$$

wherefrom

$$
\begin{gathered}
\sin \widetilde{\alpha}_{f}=\frac{d_{2}-2 l_{1} \sin \alpha \sin \left(\frac{\theta}{2}\right)}{R_{1}}=-\sin \alpha_{0}, \\
d_{2}-2 l_{1} \sin \left(\beta_{0}+\frac{\theta}{2}\right) \sin \left(\frac{\theta}{2}\right)=-d_{2}, \\
2 d_{2}=l_{1}\left[\cos \beta_{0}-\cos \left(\beta_{0}+\theta\right)\right], \\
\theta_{f}=\arccos \left(\frac{l_{1} \cos \beta_{0}-2 d_{2}}{l_{1}}\right)-\beta_{0}, \\
\theta_{f}=\arccos \left(\frac{d_{1}-d_{2}}{l_{1}}\right)-\beta_{0} .
\end{gathered}
$$

An iterative algorithm may be developed in order to determine the distance $d$. If the maximum displacement of the valve $s_{\max }$ is known, then we follow the steps:

1. consider $d_{2}=\left(d_{2}\right)_{0}$ as initial value;

2. calculate in order $\beta_{0}, \tilde{\alpha}_{0}, \theta_{f}, \alpha, s$, the deviation $\Delta$ with the formula $\Delta=s-s_{\max }$;

3. if $|\Delta|>\varepsilon$, where $\varepsilon$ is the a priori imposed error, we consider a new value for $d_{2}$, $d_{2} \mapsto d_{2}+$ increment, and repeat the step 2; otherwise the distance $d_{2}$ was determined.

Finally, $\beta_{f}=\beta_{0}+2 \arcsin \left(\frac{\sqrt{4 d_{2}^{2}+s_{\max }^{2}}}{2 l_{1}}\right)$.
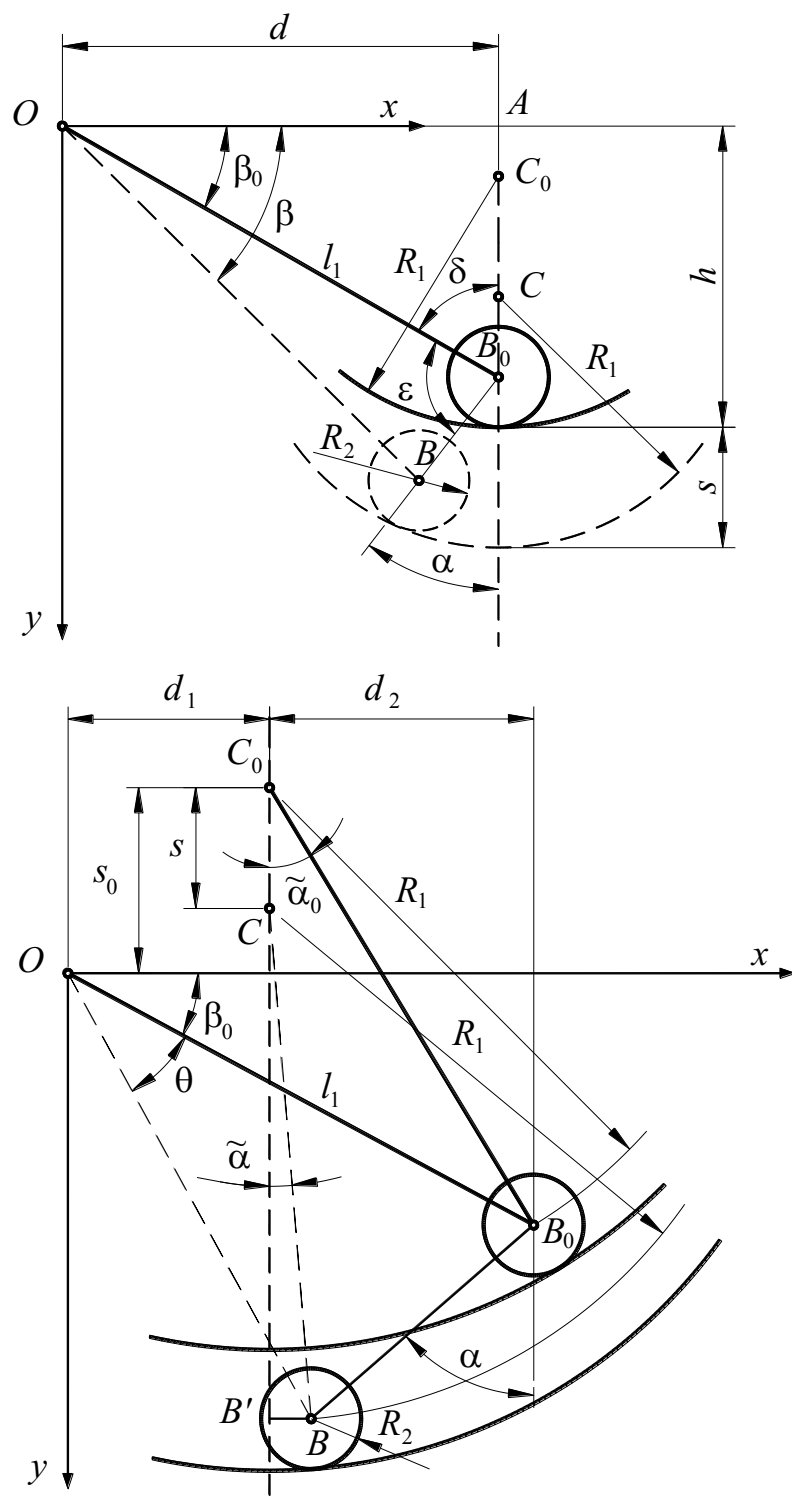

Fig. 2. The mechanism in the symmetric case.

The cases iii) and iv) are characterized by simpler formulae (Figs. 3 and 4)

$$
\begin{gathered}
\beta=\arcsin \left(\frac{s+l_{1} \sin \beta_{0}}{l_{1}}\right) \\
\theta=\beta-\beta_{0}=\arcsin \left(\frac{s+l_{1} \sin \beta_{0}}{l_{1}}\right)-\beta_{0}
\end{gathered}
$$

and

$$
\beta=\arcsin \left(\sin \beta_{0}+\frac{s}{l_{1}}\right)
$$

respectively.

At the repose position (Fig. 5) one may write the following relations [11] 


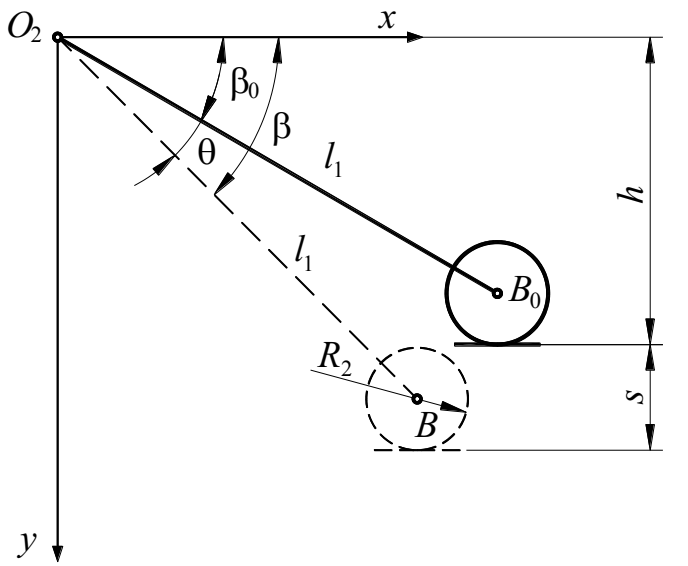

Fig. 3. The asymmetric case of planar head of valve.
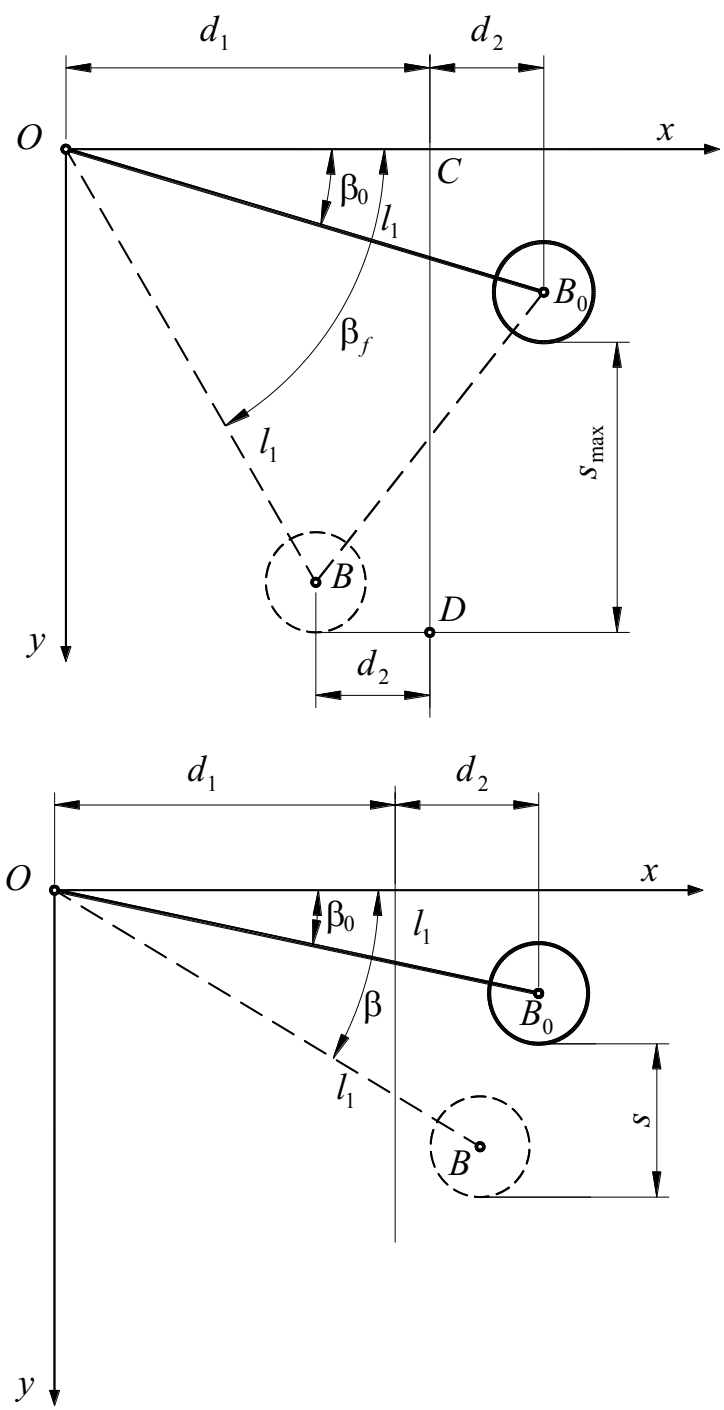

a)

b)

Fig. 4. The symmetric case of planar head of valve: $a$ ) extreme position; $b$ ) intermediate position.

$$
\begin{gathered}
\widetilde{\beta}_{0}=\pi-\beta_{0}-\delta_{0}, \\
\gamma=\arccos \left(\frac{l_{2}^{2}+d^{2}-\left(R_{0}+R_{3}\right)^{2}}{2 l_{2} d}\right),
\end{gathered}
$$

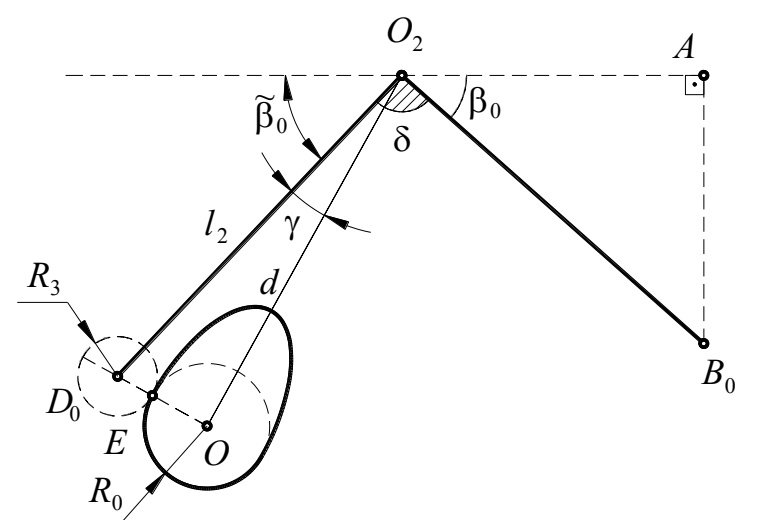

a)

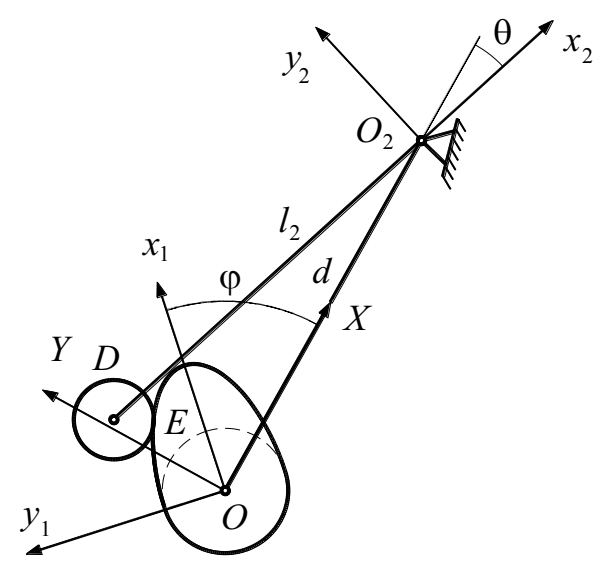

b)

Fig. 5. The synthesis of the cam: $a$ ) repose position; $b$ ) current position.

The parametric equations of the cam are $[10,11]$

$$
\begin{aligned}
x_{1} & =d \cos \varphi-l_{2} \cos (\theta+\gamma+\varphi) \\
& -R_{3} \sin (\theta+\gamma+\varphi+\lambda), \\
y_{1}= & -d \sin \varphi+l_{2} \sin (\theta+\gamma+\varphi) \\
& -R_{3} \cos (\theta+\gamma+\varphi+\lambda),
\end{aligned}
$$

where

$$
\tan \lambda=\frac{d \cos (\theta+\gamma)-l_{2}\left(1+\frac{\mathrm{d} \theta}{\mathrm{d} \varphi}\right)}{d \sin (\theta+\gamma)} .
$$

The resulted cam is not always a convex one. Considering an incremental step $\Delta \varphi$ for the rotational angle of the cam, one obtains a table of values $x_{1}\left(\varphi_{j}\right)$ and $y_{1}\left(\varphi_{j}\right)$, where $\varphi_{j}=\varphi_{0}+j \Delta \varphi$. From these values, the Jarvis March selects only those that define the convex covering of the cam. In this way, only a part of the characteristic points are selected and the cam obtained by Jarvis March contains segments of straight lines corresponding to more than one step $\Delta \varphi$.

\section{Analysis of cam}

From the equations

$$
x_{2}=-l_{2}-R_{3} \sin \lambda, y_{2}=-R_{3} \cos \lambda
$$




$$
\frac{\mathrm{d} y_{2}}{\mathrm{~d} x_{2}}=-\tan \lambda
$$

The tangency condition leads to

$$
\begin{gathered}
\tan (\varphi+\theta+\gamma)=\frac{-\frac{\mathrm{d} y_{1}}{\mathrm{~d} x_{1}}+\frac{\mathrm{d} y_{2}}{\mathrm{~d} x_{2}}}{1+\frac{\mathrm{d} y_{1}}{\mathrm{~d} x_{1}} \frac{\mathrm{d} y_{2}}{\mathrm{~d} x_{2}}} \\
=\frac{-\frac{\mathrm{d} y_{1}}{\mathrm{~d} x_{1}}-\tan \lambda}{1--\frac{\mathrm{d} y_{1}}{\mathrm{~d} x_{1}} \tan \lambda},
\end{gathered}
$$

wherefrom we obtain the expression

$$
\begin{gathered}
f_{1}(\varphi, \theta, \lambda)=\sin (\varphi+\theta+\lambda) \\
\times\left(\cos \lambda-\frac{\mathrm{d} y_{1}}{\mathrm{~d} x_{1}} \sin \lambda\right)+\cos (\varphi+\theta+\lambda) \\
\times\left(\cos \lambda \frac{\mathrm{d} y_{1}}{\mathrm{~d} x_{1}}+\sin \lambda\right)=0 .
\end{gathered}
$$

Another two functions are obtained from the contact point's coordinates, that is,

$$
\begin{gathered}
f_{2}(\varphi, \theta, \lambda)=-x_{1} \cos \varphi+y_{1} \sin \varphi+d \\
-l_{2} \cos (\theta+\gamma)-R_{3} \sin (\theta+\gamma+\lambda)=0, \\
f_{3}(\varphi \cdot \theta, \lambda)=-x_{1} \sin \varphi-y_{1} \cos \varphi \\
+l_{2} \sin (\theta+\gamma)-R_{3} \cos (\theta+\gamma+\lambda)=0 .
\end{gathered}
$$

One has to solve a nonlinear system of three equations $f_{i}=0$, with three unknowns $\varphi, \theta$, and $\lambda$. This system is solved using the Newton method, starting with the initial values $\varphi_{0}=0, \theta_{0}=0$, and $\lambda=\lambda_{0}$, where $\lambda_{0}=\arctan \left(\frac{d \cos \gamma-l_{2}}{d \sin \gamma}\right)$.

\section{Fourier analysis}

We denote by $\bar{\omega}$ the angular velocity of the cam-shaft. In these conditions, one may write

$$
\begin{gathered}
s(\varphi)=s(\bar{\omega} t) \\
=\sum_{i=1}^{\infty} a_{i} \cos (i \bar{\omega} t)+\sum_{i=1}^{\infty} b_{i} \sin (i \bar{\omega} t),
\end{gathered}
$$

where $s$ is the displacement of the valve, while $a_{i}$ and $b_{i}$ are the Fourier coefficients.

The acceleration of the valve has the expression

$$
\begin{aligned}
a(\varphi)= & a(\bar{\omega} t)=-\sum_{i=1}^{\infty} a_{i}(i \bar{\omega})^{2} \cos (i \bar{\omega} t) \\
& -\sum_{i=1}^{\infty} b_{i}(i \bar{\omega})^{2} \sin (i \bar{\omega} t),
\end{aligned}
$$

while the magnitude of the inertial force is

$$
F(\varphi)=F(\bar{\omega} t)=m_{e q} a(\varphi),
$$

$m_{e q}$ being the equivalent mass of the distribution mechanism.

The force induced by the $j$ th valve reads

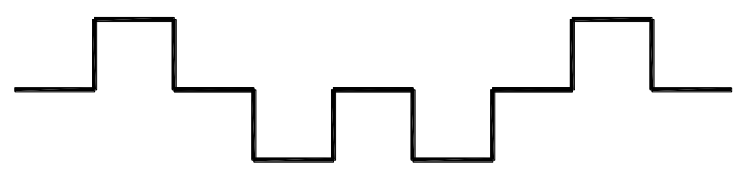

$1-3-4-2$
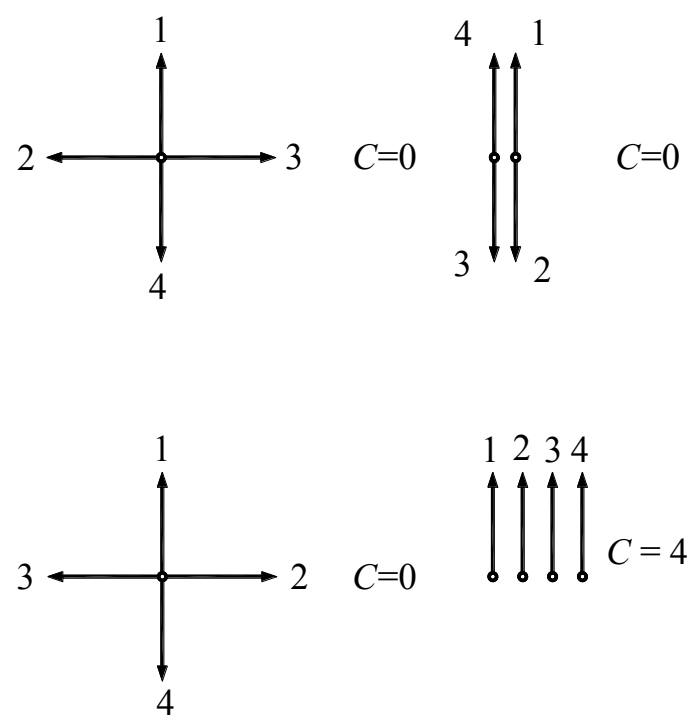

Fig. 6. The cam-star for the Fourier analysis.

$$
F_{i j}(\bar{\omega} t)=-m_{e q} A_{i}(i \bar{\omega})^{2} \cos \left(i\left(\bar{\omega} t+\gamma_{j}+\theta\right)\right) ;
$$

the force induced by all four valves (we assumed a fourstroke engine with 4 cylinders, the firing order being 1 4-3-2 (Fig. 6)) is

$$
F_{i}(\bar{\omega} t)=-m_{e q} A_{i}(i \bar{\omega})^{2} \sum_{j=1}^{4} \cos \left(i\left(\bar{\omega} t+\gamma_{j}+\theta\right)\right),
$$

and the net inertial force is given by

$$
\sum_{j=1}^{4} \cos \left(i\left(\bar{\omega} t+\gamma_{j}+\theta\right)\right)=C_{i} \cos \left(i\left(\bar{\omega} t+\gamma_{j}+\theta\right)\right) \text {. }
$$

Analyzing Fig. 6, we may conclude the most important coefficients are $C_{4 k}=4, k$ integer, the rest of coefficients being zero.

It results the magnitude of the inertial force and the amplitude of oscillations as

$$
\hat{F}_{i}=4 A_{i} m_{e q}(4 k \bar{\omega})^{2}, \hat{A}_{i}=\frac{\hat{F}_{i}}{M_{e}(i \bar{\omega})^{2}},
$$

$M_{e}$ being the mass of the engine.

One may write the following relations

$$
\begin{gathered}
\left\{\varphi_{i}\right\}=\{0, \pi, \pi, 0\} \\
\left\{\gamma_{j}\right\}=\left\{0, \frac{\pi}{2}, \frac{3 \pi}{2}, \pi\right\} \\
i=1 \Rightarrow\left\{i \gamma_{j}\right\}=\left\{0, \frac{\pi}{2}, \frac{3 \pi}{2}, \pi\right\} \\
i=2 \Rightarrow\left\{i \gamma_{j}\right\}=\{0, \pi, 3 \pi, 2 \pi=0\} \\
i=3 \Rightarrow\left\{i \gamma_{j}\right\} \\
=\left\{0, \frac{3 \pi}{2}, \frac{9 \pi}{2}=\frac{\pi}{2}, 3 \pi=\pi\right\},
\end{gathered}
$$




$$
\begin{gathered}
i=4 \Rightarrow\left\{i \gamma_{j}\right\} \\
=\{0,2 \pi=0,6 \pi=0,4 \pi=0\} .
\end{gathered}
$$

\section{Numerical simulation}

The following values were selected for the numerical simulation: $\quad l_{1}=0.01612 \mathrm{~m}, \quad R_{1}=0.025 \mathrm{~m}$, $R_{2}=0.010 \mathrm{~m}, \quad \beta_{0}=15^{0}, \quad s_{\max }=0.00935 \mathrm{~m}$, $R_{0}=0.018 \mathrm{~m}, \quad d=0.042 \mathrm{~m}, \quad l_{2}=0.060 \mathrm{~m}$, $R_{3}=0.01867 \mathrm{~m}, d_{1}=0.010 \mathrm{~m}$, the angles $\varphi_{1}=92^{0}$ (valve starts to open), $\varphi_{2}=161^{0}$ (valve is completely open), $\varphi_{3}=180^{\circ}$ (valve starts to close), and $\varphi_{4}=244^{\circ}$ (valve is completely closed). Two laws of motion are considered for the valve displacement [9-11]

$$
\begin{gathered}
s_{1}(\varphi)= \\
0, \varphi \in\left[0, \varphi_{1}\right), \\
s_{\max } \sin ^{2}\left(\frac{90\left(\varphi-\varphi_{1}\right)}{\varphi_{2}-\varphi_{1}}\right), \varphi \in\left[\varphi_{1}, \varphi_{2}\right], \\
s_{\max } \sin ^{2}\left(\frac{90\left(\varphi+\varphi_{4}-2 \varphi_{3}\right)}{\left.\varphi_{4}-\varphi_{3}\right)}\right), \varphi \in\left[\varphi_{3}, \varphi_{4}\right], \\
0, \varphi \in\left(\varphi_{4}, 360\right] \\
s_{2}(\varphi)= \\
0, \varphi \in\left[0, \varphi_{1}\right), \\
s_{\max } \frac{\left(\varphi-\varphi_{1}\right)^{2}\left(\varphi-2 \varphi_{2}+\varphi_{1}\right)^{2}}{\left(\varphi_{2}-\varphi_{1}\right)^{4}}, \varphi \in\left[\varphi_{1}, \varphi_{2}\right], \\
s_{\max }, \varphi \in\left(\varphi_{2}, \varphi_{3}\right), \\
s_{\max } \frac{\left(\varphi-\varphi_{4}\right)^{2}\left(\varphi-2 \varphi_{3}+\varphi_{4}\right)^{2}}{\left(\varphi_{3}-\varphi_{4}\right)^{4}}, \varphi \in\left[\varphi_{3}, \varphi_{4}\right], \\
0, \varphi \in\left(\varphi_{4}, 360\right] .
\end{gathered}
$$

The resulted cams are given in Fig. 7 .

In Fig. 8 are drawn the real laws of motion for the normal cams, and the laws of motion for the convex cams in some cases.

Some Fourier coefficients $a_{i}$ are given in Table 1.

a)

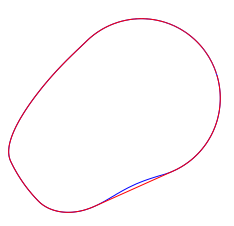

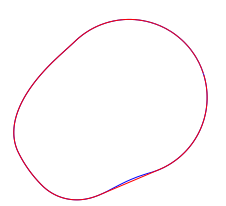

b)

c)

d)

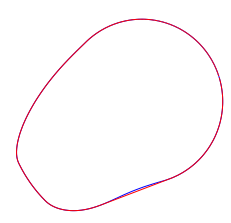

e)

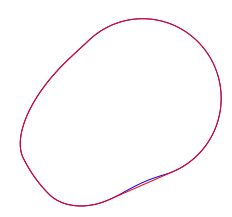

f)
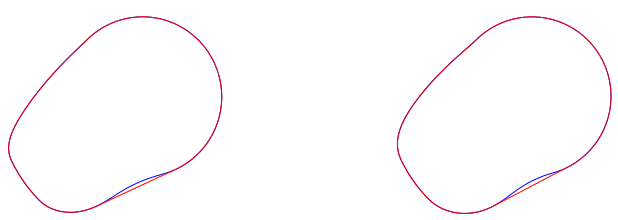

$g)$

h)

Fig. 7. The obtained cam for: $a$ ) case i) first law; $b$ ) case i) second law; $c$ ) case ii) first law; $d$ ) case ii) second law; $e$ ) case iii) first law; $f$ ) case iii) second law; $g$ ) case iv) first law; $h$ ) case iv) second law. The normal cam in drawn in blue, the convex one obtained with the aid of Jarvis March in red.
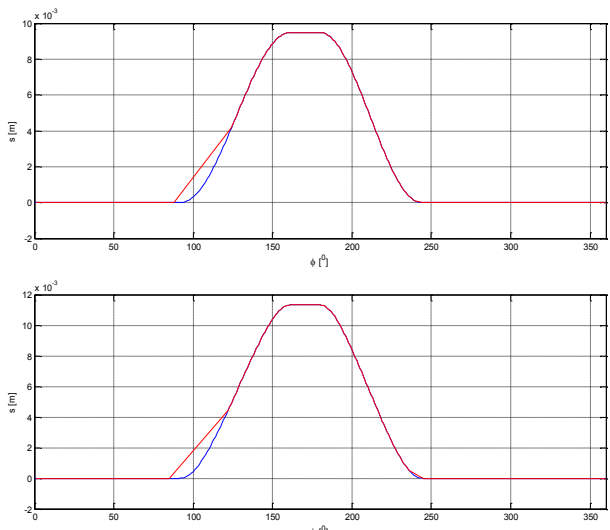

a)

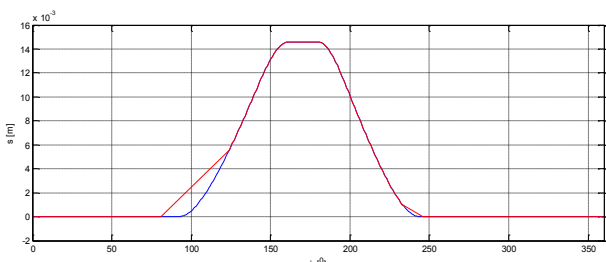

b)

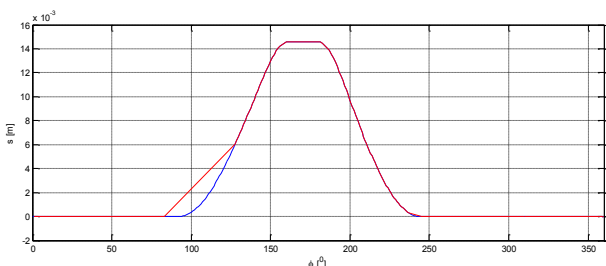

c)

Fig. 8. The laws of motion for the real cams (blue) and convex cams (red) for: $a$ ) case i) first law; $b$ ) case iii) second law; $c$ ) case ii) second law; $d$ ) case iv) first law.

One may write the following observations:

- symmetric case leads to bigger cams;

- the two laws of motion lead to similar cams;

- the obtained cams are not convex and the Jarvis March must be applied;

- the coefficient $a_{4}$ is the most important one.

The Fourier coefficients $b_{i}$ are not presented in this paper, but for non-symmetric angles of opening and closing of valves these coefficients do not vanish. 
Table 1. Fourier coefficients in some cases.

\begin{tabular}{|c|c|c|c|c|}
\hline Law & \multicolumn{4}{|c|}{ First } \\
\hline Case & i) & i) Jarvis & ii) & ii) Jarvis \\
\hline$a_{0}$ & 0.0044413 & 0.0046178 & 0.0044413 & 0.0064828 \\
\hline$a_{1}$ & 0.0038423 & 0.0038930 & 0.0038423 & 0.0054888 \\
\hline$a_{2}$ & 0.0024231 & 0.0022955 & 0.0024231 & 0.0033474 \\
\hline$a_{3}$ & 0.0009880 & 0.0008999 & 0.0009880 & 0.0015438 \\
\hline$a_{4}$ & 0.0001240 & 0.0002080 & 0.0001240 & 0.0006347 \\
\hline$a_{8}$ & -0.0000165 & -0.0000294 & -0.0000165 & -0.0000505 \\
\hline$a_{12}$ & 0.0000015 & -0.0000083 & 0.0000015 & 0.0000335 \\
\hline$a_{16}$ & 0.0000059 & 0.0000190 & 0.0000059 & 0.0000222 \\
\hline$a_{20}$ & 0.0000009 & 0.0000060 & 0.0000009 & -0.0000187 \\
\hline Law & \multicolumn{4}{|c|}{ Second } \\
\hline Case & iii) & iii) Jarvis & iv) & iv) Jarvis \\
\hline$a_{0}$ & 0.0046717 & 0.0054925 & 0.0046717 & 0.0069123 \\
\hline$a_{1}$ & 0.0039965 & 0.0045939 & 0.0039965 & 0.0057659 \\
\hline$a_{2}$ & 0.0024178 & 0.0026665 & 0.0024178 & 0.0033530 \\
\hline$a_{3}$ & 0.0008729 & 0.0010631 & 0.0008729 & 0.0014174 \\
\hline$a_{4}$ & 0.0000108 & 0.0002919 & 0.0000108 & 0.0005069 \\
\hline$a_{8}$ & -0.0000149 & -0.0000006 & -0.0000149 & -0.0000112 \\
\hline$a_{12}$ & -0.0000039 & 0.0000073 & -0.0000039 & 0.0000093 \\
\hline$a_{16}$ & 0.0000066 & 0.0000168 & 0.0000066 & -0.0000002 \\
\hline$a_{20}$ & 0.0000019 & -0.0000061 & 0.0000019 & -0.0000234 \\
\hline
\end{tabular}

\section{Conclusions}

Some conclusions can be drawn:
- the Miller-Atkinson cycle does not lead to convex cams and the Jarvis March must be applied. Some simulations for larger radii for the base circle were performed, but, for acceptable radii the cams are not convex;

- the Fourier coefficients $a_{i}$ are greater for the convex cam;

- the non-convexity of the cam is mainly caused by the opening of the valve.

The influence of different parameters on the dynamics of the mechanism will be the goal of our future work.

\section{References}

1. G. Gonca, B. Sahin, A. Parlak, Y. Ust, V. Ayhan, I. Cesur, B. Boru, Applied Energy 138, 11 (2015)

2. M.E.S. Martins, T.D.M. Lanzanova, Applied Thermal Engineering 90274 (2015)

3. R. Ebrahimi, Mathematical and Computer Modelling 53, 1289 (2011)

4. J.P. Zammit, M.J. McGhee, P.J. Shayler, T. Lawa, I. Pegg, Energy 79, 100 (2015)

5. Y. Murata, J. Kusaka, M. Odaka, Y. Daisho, D. Kawano, H. Suzuki, H. Ishii, Y. Goto, SAE paper 2006-01-0203 (2006)

6. W. De Ojeda, SAE paper 2010-01-1124 (2010)

7. A. Kéromnčs, B. Delaporte, G. Schmitz, L. Le Moyne, Energy Conversion and Management 82, 259 (2014)

8. S. Zhu, K. Deng, S. Liu, S. Qu, Energy 93, 75 (2015)

9. I. Dragomir, B. Mănescu, N.-D. Stănescu, AVMS 2017, Timișoara (2017)

10. I. Dragomir, B. Mănescu, N.-D. Stănescu, N. Pandrea, ICMSAV XLI, Cluj-Napoca (2017)

11. I. Dragomir, B. Mănescu, N.-D. Stănescu, N. Pandrea, A. Clenci, D. Popa, CAR 2017, Pitești (2017)

12. B. Yan, H. Wang, Z. Zheng, Y. Qin, M. Yao, Applied Thermal Engineering 122, 439 (2017)

13. B. Wu, Q. Zhan, X. Yu, G. Lv, X. Nie, S. Liu, Applied Thermal Engineering 118, 621 (2017)

14. J. Zhao, Applied Energy 185, 300 (2017)

15. T. Li, B. Wang, B. Zheng, Energy Conversion and Management 123, 140 (2016)

16. Q. Luo, B. Sun, Energy Conversion and Management 123, 209 (2016)

17. G. Gonca, Energy 109, 152 (2016)

18. Y. Wang, B. Zu, Y. Xu, Z. Wang, J. Liu, Energy Conversion and Management 119, 316 (2016)

19. E. Dobrucali, Energy 103, 119 (2016)

20. G. Gonca, B. Sahin, Applied Mathematical Modelling 40, 3764 (2016) 